\title{
Development and preliminary testing of a framework for quantifying local service provision for people with dementia
}

\begin{abstract}
Purpose

It was hypothesised that there were variations in health and social care services available for older people with dementia and their carers and that measurement of this between localities was possible. The purpose of this paper is to present a framework for examining this.
\end{abstract}

Design/methodology/approach

Using a case study approach, data from national surveys of local authorities providing social care and NHS Trusts providing old age mental health services conducted in 2014/5 in England were used. From these, indicators of variation in services for people with dementia and their carers in different geographical areas were created. Measurement of the presence/absence of each service permitted the creation of a service mix score for each area.

\section{Findings}

The framework comprised 16 attributes each with indicators describing the characteristics of the organisations providing the services; the skill mix of community mental health teams for older people; and the healthcare and social care services available in localities. Variation was evident, confirmed by quartile analysis and exemplars, suggesting that older people with dementia and their carers in different localities are likely to experience differences in the range of provision available, particularly social care services.

Originality/value

The case study approach employed achieved its objectives and the resultant framework has potential for generalisability and utility given acceptable ecological validity and discriminant validity in identifying variations in service mix. It could be utilised in both research and practice.

Classification: Research paper

Key words: Social care, Healthcare, Framework, Older people with mental health problems, Carers

\section{Funding information}

Programme Grants for Applied Research, Grant/Award Number: DTC-RP-PG-031112003; National Institute for Health Research (NIHR), England 


\section{Introduction}

Supporting people with dementia at home is a longstanding international challenge (Alzheimer's Disease International, 2020). Home support is characterised by a range of services, which complement informal support provided by family and friends, described as carers. These services are usually categorised according to purpose and include: supportive, emotional and practical help; education, including skills training in managing behaviour; and more structured therapeutic interventions, such as counselling. In England, the second half of the $20^{\text {th }}$ century witnessed the growth of community care for older people with dementia with services transferred from inpatient health services to enable people to live at home (Cmnd 1604, 1962; Cmnd 6233, 1975; $\mathrm{Cm}$ 849, 1989). These services are commissioned by the National Health Services and local authorities, the latter being geographical areas and units of local government responsible for the provision of social care. Increasinlgy services are jointly commissioned by these two bodies (Department of Health and Social Care, 2018)

The importance of a diverse infrastructure of health and social care services within a community to allow people with dementia to live at home as an alternative to long-term care has long been recognised (Challis et al., 2016; Gladman et al., 2007). However, little is known about the range of services available within geographical areas and the extent to which they differ across the country. The aim of this paper is to describe the development of a framework for examining variation in health and social care services available to support older people with dementia and their carers at home. A case study approach was employed here, to explore the complexities, circumstances, and range of services provided in localities, using the country, (England) as the unit of analysis (Yin, 1984). This has been recognised as a valuable method for the study of complex settings in the early stages of research and for the generation of hypotheses (Bowling, 2014). It was hypothesised that there were:

(i) Variations in health and social care arrangements for older people with dementia, and

(ii) It was possible to measure the extent of variation between localities (geographical areas).

A conceptual framework to explain the relationship between the main variables in the study, local health and social care services to support older people with dementia and their carers at home was developed to explore these hypotheses further (Adom et al., 2018).This study was one of nine within a research programme entitled, 'Effective Home Support in Dementia Care: Components, Impacts and Costs of Tertiary Prevention'.

\section{Method}

Approval for this study was obtained from the University of Manchester Ethics Committee on 24th June 2014 (ref: 14209).

\section{Data collection}

Data for this study was obtained from two parallel surveys: a national survey of local authorities providing social care and a national survey of NHS Trusts providing old age 
mental health service. The first survey, to local authorities, investigated services for people aged 65 and over living in the community, particularly those exclusively for older people with mental health problems. There were five foci of enquiry: home care provision; enablement services; respite care arrangements; residential and nursing care provision; and arrangements for joint commissioning with local NHS commissioners (Davies et al., 2019). The second survey explored the range and scope of NHS Trust community provision for people with dementia. There were three foci of enquiry: commissioning arrangements; memory clinics; and community mental health teams (CMHTs) for older people (Ahmed et al., 2018) . A subset of data from these two surveys were used in this analysis.

The survey of local authority managers employed a phased mail out to facilitate the identification of NHS Trusts providing complementary services for older people with dementia in the same locality. Data collection commenced in late 2014 and continued until the end of 2015. With Trusts identified, data collection in these took place in 2015. Copies of the questionnaire were both posted and emailed to local authority and Trust managers. There were 122 completed returns from the local authority survey, a response rate of 83 per cent. Individual NHS Trusts provide old age mental health services for a number of local authority areas. For the NHS survey, data were obtained from 51 of the 60 NHS Trusts, a response rate of 85 per cent. These provided data relating to $120(79 \%)$ local authority areas. From both sets of returns, a matched dataset was created providing details of health and social care services in 96 geographical areas.

\section{Data analysis}

The data from the questionnaires were entered into an SPSS database (IBM SPSS Statistics version 22). Descriptive statistics were used to report findings from the surveys concerning the indicators of home support within localities. Subsequently, analysis was undertaken to create a service mix score, representing the potential for users and carers resident in a particular area to receive a range of services.

\section{Identification of indicators}

A staged approach was used to create indicators of variation in services for people with dementia and their carers: (1) testing the concept; (2) exploration of the range of items available; and (3) selection and refinement of the items for inclusion in a service mix score.

In stage one, the feasibility of the concept was tested by appraising the viability of selecting items from survey returns to identify differences in service provision between localities. A sample consisting of the first 50 local authority returns was used from which seven items was selected. Data were extracted to explore differences in home care availability ( 3 items), staff-mix in CMHTs, activities undertaken by CMHTs, availability of respite care and occupational therapy. The range of services available for older people with dementia in a geographical area was calculated from the summation of these items and ranked from high to low. It was hypothesised that this variability would be replicated in a larger sample.

Consequently, in stage two, the range of items available from both the local authority and NHS surveys was explored. Some items were excluded due to missing values. 
Nine items were added from the local authority survey to those identified in stage 1. These 16 items were summed and ranked from high to low. This provided further evidence of variability within the dataset. Subsequently, the first 50 NHS returns were used to select a further ten items. At the end of stage 2 it was concluded that these 26 items from the local authority and NHS surveys gave an indication of the range of services which might be available in a geographical area.

In stage three, the items identified in stage two were refined using a matched dataset, with data available from both local authority and NHS respondents for a geographical area $(n=43)$. Items were selected based on the requirements of face validity and discriminatory power. Some items from the NHS survey were excluded because they did not have sufficient variability, for example the presence of old age psychiatrist in a $\mathrm{CMHT}$ was noted in all returns. Another relating to the size of CMHT for older people was excluded because it lacked face validity. This refinement led to the creation of a final list of 16 indicators including three composite items reflecting NHS services for people with dementia and their carers relating to early stage, later stage and services specifically for carers.

\section{Service mix score}

A service mix score was created to measure the presence/absence of each of the 16 framework indicators for each geographical area. It was designed to represent the potential or capacity for users, resident in that locality, to receive a range of different services. This permitted geographical areas to be placed in rank order with quartile divisions used to create groupings by score. Additionally, the distributional form of the score, in terms of the number of geographical areas scoring on each point of the scale, was tested. Testing was undertaken through the software package EasyFitXL (Mathwave, 2017).

\section{Results}

\section{Service framework}

The final list of 16 indicators from the analysis is listed in Table 1. These are described under four broad headings. Firstly, there are indicators describing the characteristics of the organisations providing the services. These discriminate whether they were jointly specified by health and social care commissioners and whether or not they were provided by a single health and social care provider. Second, the service characteristics of CMHTs are described in terms of their skill mix and specifically whether or not they included a social worker, generic support worker, occupational therapist assistant or Admiral Nurse. (The latter are practitioners providing support primarily to the families of people with dementia.) Third, healthcare services are categorised in terms of those focusing primarily on early stage dementia, later stage dementia and support to carers. (Details of these are provided as a footnote to the tables). Fourth, there are indicators of local authority social care services. Two relate to home care (specialist provision for people with dementia and night time availability). Three denote respite services (specialist overnight or day care for people with dementia or placement within a family). Specialist hospital discharge services and assistive technologies for people with dementia complete the list of social care indicators. 
Fourth, there are indicators of local authority social care services. These consist of two relating to home care (specialist provision for people with dementia and night time availability); respite services (specialist overnight or day care for people with dementia or placement within a family); and specialist hospital discharge services and assistive technologies for people with dementia.

\section{Capturing differences between geographical areas}

The service mix score, derived from the indicators, ranged from 0 to 16. Its distribution across geographical areas is illustrated in Figure 1. No geographical areas scored the maximum of 16 . The minimum score was three exhibited by only one geographical area. Twenty three (24\%) localities within the lower quartile had a service mix score of 7 and under; 24 (25\%) localities within the upper quartile had a score of 11 or higher. The distribution tests conducted on the data showed that the service mix score fitted a normal distribution (Kolmogorov-Smirnov Test, 0.15469; $\mathrm{p}=0.017$ ), with a mean of $8.9(\mathrm{SD}=2.34)$.

Measures of variation are shown in Table 2. It shows the percentage of geographical areas for each indictor by interquartile range. Unsurprisingly, there was an upward trend for geographical areas to display more positive responses to indicators across the range. Most $(n=14)$ of the indicators showed increases across the inter quartile ranges. For two of the indicators there were a high proportion of geographical areas responding positively in all three quartiles. These were 'the availability of home care at night' and 'specialist overnight respite care for people with dementia'. No geographical areas in the lower quartile reported the presence the indicator, 'Admiral Nurse'. In contrast, the indicator of 'specialist day care' was present in all geographical areas in the upper quartile. Within quartile variation is also apparent in Table 2, in terms of the number of geographical areas with particular indicators. However, this variation is less in the upper quartile compared with the other two.

Table 3 provides evidence of the practical application of the service mix score. It provides exemplars of indicators reported within geographical areas with a low (Area $A$ ), medium (Area B) and high (Area $C$ ) range of services. From a maximum of 16 indicators, seven, nine and twelve respectively are recorded as present in the geographical areas represented by the data. The principal difference between the area with a low range of services (Area A) compared with the other two is the small number of social care services for people with dementia and their carers. In contrast, the exemplar with the highest number of services (Area $\mathrm{C}$ ) had more services provided by the NHS than the other two.

\section{Discussion}

The analysis in this paper explored two hypotheses, that there were variations in health and social care arrangements for older people with dementia, and that it was possible to measure the extent of variation between localities. This was achieved through the development of a framework detailing health and social care services available to support people at home using data from two national surveys of service provision. Variations in both the number and types of service available in England were evident. However, there were inevitably limtations to this approach. Firstly, 
these data provided a snapshot of NHS and local authority service provision at a point in time. Such cross-sectional surveys do not provide robust evidence about the direction of service development or the cost of services. Secondly, the framework was derived from data representing health and social care service configurations in two thirds of geographical areas in England. However, service configurations and practice are predicated on national guidance indicating a more general applicability (Department of Health and Social Care, 2018; Department of Health 2009). Thirdly, the development of the framework was constrained by the requirement for indicators to demonstrate evidence of variability and the capacity to measure their presence or absence. Fourthly, the service mix framework does not include information about providers of social care older people with dementia their carers. However, there is evidence that this is increasingly provided by the non-statutory (for-profit, not-for-profit, voluntary) sector (Davies et al., 2020; Jasper et al., 2016). Below the framework developed in this paper is appraised from three perspectives.

\section{Ecological validity}

A strength of this study was that it sought to achieve 'ecological validity', in making the data fit the real world of service provision (Banister et al. 1994 p. 5). This was achieved through the use of primary data collected from local authorities and the NHS as the the basis for the development of the indicators. These provided data relating to 120 geographical areas, 79 per cent of English local authorities. In the language of performance management, these data can be characterised as supply and service process indicators (Challis et al., 2016); they are a mixture of staffing, service provision and organisational structure. Supply indicators relate to what is available and refer to the service system in a broad sense, whereas service process indicators cover the extensiveness and mix of provision. They represent a bottom up perspective in that they are derived from surveys describing provision at a point in time. In tandem, we think these indicators represent prescribed services that individuals may experience which might lead to valued outcomes from the perspective of service users and carers. Thus, these differ from other nationally available measures of process and outcome (Department of Health, 2016).

\section{Spectrum of services}

Data, in Tables 2 and 3, reveal variation in services reflecting that national uniformity drives the NHS and local diversity in local authorities (Wistow, 2012). Seven indicators within the framework relate to social care services and Table 2 provides evidence of their variation between geographical areas. We surmise that our data also show that three services funded by local authorities - home care night time, specialist overnight respite care and specialist day care - might be regarded as core services given their prominence in the national survey (Table 1) and in the exemplars in Table 3 . The availability of assistive technologies specific to the needs of older people with dementia might also be added to this list (Table 1 and Table 2).

It has recently been estimated that there are likely to be over 200,000 new dementia cases per year in the UK. This coupled with increased longevity means that people with dementia in the oldest age groups are likely also to exhibit physical frailty (Matthews et al., 2016). Appropriate care for people with dementia to enable them to live at home is therefore likely to require substantial service inputs to compensate for 
reduced abilities to undertake activities of daily living and instrumental activities of daily living. This will be additional to the support provided by carers who are likely to have their own needs in this respect. Given this uniformity of need we were not clear whether the question becomes the extent to which variance across the country reflects local needs or, more negatively, service deficits in certain areas of the country. Carers of people with dementia value respite services (Newbronner et al., 2013) therefore we think variability in provision highlighted in our data is of particular concern.

\section{Application}

This case study has provided an insight into service arrangements for people with dementia and their carers in England. It incorporates indicators relating to both health and social care services. This framework could, for example, provide commissioners with information to develop service specifications, a means of promoting quality assurance within service providers and of monitoring the latter's performance. It also has the capacity to provide a vehicle for communication between commissioners and providers to promote continuous improvement. In these unprecedented times in the wake of the COVID 19 pandemic we think it has the potential to maintain a focus on the provision of services for older people with dementia and their carers as a priority (Alzheimer's Disease International, 2020). Furthermore, it complements data already available for older people with dementia. Whilst there are tools to improve and monitor old age mental health services provided by the NHS they do not extend to social care services or those which are jointly provided (Royal College of Psychiatrists, 2018; 2019). There is other, predominantly clinical, information which maps provision in England relating to five objectives (preventing well, diagnosing well, supporting well, living well, and dying well) (Department of Health, 2016). The information relating to social care in this data set is small and confined to that provided by the voluntary sector.

The utility of the framework to inform further research is emerging. In one study underway (Chester et al., 2017), to explore the presence, effects, and costeffectiveness of approaches to home support for people in later stage dementia and their carers, it has been used to select fieldwork sites. This will permit comparisons of individual experiences in areas with demonstrably different service configurations. The framework for example permits sites to be stratified into three groups (low 1-8, medium 9-10 and high 11 or more) using the indicators. Furthermore, the distribution tests conducted on the framework data, reported above, show that the service mix score fits a normal distribution. This would enable the score to be used appropriately in future multivariate analysis, examining what might predict local areas' potential mix of dementia services. Furthermore, we think such an approach could also examine the links between service availability and potential outcomes for individuals in geographical areas with different ranges of provision.

\section{Conclusion}

From this study we may conclude that the case study approach employed achieved its objectives. Furthermore, we believe the conceptual framework we developed has promoted transparency in the identification and measurement of health and social care services in localities thereby generating a means by which variation may be measured. Interestingly this logical approach offers a framework with potential for generalisability 
and utility given acceptable ecological validity, discriminant validity in identifying variations in service mix and utility in areas of potential application for both research and practice.

\section{Funding}

This article presents independent research funded by the National Institute for Health Research (NIHR). The views expressed are those of the authors and not necessarily those of the NIHR or the Department of Health and Social Care.

\section{References}

Adom, D., Hussein, E. and Adu-Agyem, J. (2018) "Theoretical and conceptual framework: mandatory ingredients of a quality research", International Journal of Scientific Research, Vol., 7, No. 1, pp. 438-441.

Ahmed, S., Hughes, J., Davies, S., Stewart, K., Orrell, M., Clarkson, P., Challis, D. and Members of the HoSt-D (Home Support in Dementia) Programme Management Group (2018) "Specialist services in early diagnosis and support for older people with dementia in England: Staff roles and service mix", Interational Journal of Geriatric Psychiatry, https://doi.org/10.1002/gps.4925

Alzheimer's Disease International (2020), From Plan to Impact III: Maintaining Dementia as a Priority in Unprecedented Times, Alzheimer's Disease International, London. https://www.alz.co.uk/adi/pdf/from-plan-to-impact-2020.pdf $\quad$ (accessed $6^{\text {th }}$ October 2020)

Banister, P., Burman, E., Parker, I., Taylor, M. and Tindall, C. (1994), Qualitative Methods in Psychology: A Research Guide, Open University Press, Buckingham.

Bowling A. (2014), Research Methods in Health: Investigating Health and Health Services, Open University Press, Buckingham.

Challis, D., Clarkson, P. and Warburton, R. (2006) Performance Indicators in Social Care, Ashgate, Aldershot.

Chester,H., Clarkson, P., Hughes,J., Russell, I., Beresford, J., Davies, L., Jolley, D., Peconi, J., Poland, F., Roberts, C. Sutcliffe, C. Challis, D. and Members of the HoStD (Home Support in Dementia) Programme Management Group, (2017), "Evaluating the effectiveness of different approaches to home support for people in later stage dementia: a protocol for an observational study", International Psychogeriatrics, Vol. 29, No. 7, pp. 1213-21.

Challis, D., Sutcliffe, C., Hughes, J., von Abendorff, R., Brown, P. and Chesterman, J, (2016) Supporting People with Dementia at Home: Challenges and Opportunities for the 21st Century, Routledge, London. Second edition.

Cm 849 (1989), Caring for People: Community Care in the Next Decade and Beyond, HMSO, London. 
Cmnd 1604 (1962), A Hospital Plan for England and Wales, HMSO, London.

Cmnd 6233 (1975), Better Services for the Mentally III, HMSO, London.

Davies, K., Dalgarno, E., Angel, C., Chester, H., Jasper, R., Roberts, A. and Challis, D. (2020) "Home-care providers as collaborators in commissioning arrangements for older people", Health and Social Care in the Community, https://doi.org/10.1111/hsc. 13169

Davies, S., Hughes J., Ahmed S., Clarkson, P., Challis, D., and Members of the HoStD (Home Support in Dementia) Programme Management Group, (2019) "Commissioning social care for people with dementia living at home: Findings from a national survey", International Journal of Geriatric Psychiatry, https://doi.org/10.1002/gps.5214

Department of Health (2009), Living Well with Dementia: A National Dementia Strategy, Department of Health, London.

Department of Health, (2016), "Dementia Atlas" available at: https://shapeatlas.net/dementia/\#6/52.9448/-2.1472/I-p65. (accessed 7 December 2016).

Department of Health and Social Care, (2018), Care and Support Statutory Guidance, Department of Health and Social Care, London. (accessed 26 June 2018).

Gladman JR, Jones RG, Radford K, Walker E, Rothera, I. (2007), "Person-centred dementia services are feasible, but can they be sustained?", Age and Ageing, Jan Vol.,19. No. 36(2), pp. 171-6.

Jasper, R., Hughes, J., Sutcliffe, C., Abendstern, M., Loynes, N. and Challis, D. (2016) "Accessing care coordination information: the non-statutory sector contribution", Quality in Ageing and Older Adults, Vol.,17. No. 4, pp. 263-271.

Mathwave (2017), EasyFitXL: Distribution Fitting for Excel, Mathwave, Milpitas, California.

Matthews, F.E., Stephan, B.C.M., Robinson, L., Jagger, C., Barnes, L.E., Arthur, A., Brayne, C. and Cognitive Function and Ageing Studies (CFAS) Collaboration (2016), "A two decade dementia incidence comparison from the Cognitive Function and Ageing Studies I and II", Nature Communications, Vol. 7, 11398. https://doi.org/10.1038/ncomms 11398

Newbronner, L., Chamberlain, R., Borthwick, R., Baxter, M., and Glendinning, C. (2013), A Road Less Rocky: Supporting Carers of People with Dementia, Carers Trust, London.

Royal College of Psychiatrists (2018), "Memory Services National Accreditation Programme (MSNAP) Standards for Memory Services", 6th Edition published April available at: https://www.rcpsych.ac.uk/docs/default-source/improving- 
care/ccai/quality-networks/memory-clinics-msnap/msnap-standards-6th-edition2018.pdf?sfvrsn=d3a9bc94 2 (accessed 6th October 2020)

Royal College of Psychiatrists (2019), "Standards for Older Adult Mental Health Services", 5th Edition available at: https://www.rcpsych.ac.uk/docs/defaultsource/improving-care/ccai/quality-networks/older-adult-wards-qn/qnoamhs-5thedition-standards.pdf?sfvrsn=ac2002d4 2 (accessed 6th October 2020)

Wistow, G. (2012), "Still a fine mess? Local government and the NHS 1962 to 2012", Journal of Integrated Care, Vol. 24. No. 2, pp. 101-14.

Yin, R. (1984), Case Study Research: Design and Methods., Sage, Beverly Hills CA 